\title{
The Pain and Joy of a Major Scientific Discovery
}

\author{
R. E. Marshak ${ }^{\dagger}$ \\ Banquet Talk on the occasion of E. C.G. Sudarshan's 60th birthday celebration.
}

Z. Naturforsch. 52a, 3-8 (1997)

This three-day workshop is being held on the occasion of George Sudarshan's 60th birthday to do honor to his significant and many-faceted contributions to theoretical physics. The nature and extent of these contributions are being covered by a host of distinguished speakers and, hence, I shall limit my banquet remarks to George Sudarshan's early scientific career in particle physics which culminated in his remarkable doctoral dissertation on the nature of the four-fermion interaction, otherwise known as the universal $\mathrm{V}-\mathrm{A}$ theory of weak interactions. George Sudarshan's thesis problem turned out to be so exciting that his professor entered as a part-time participant. This was easy to do because George was brought up in the Indian tradition which instills great respect on the part of the student towards his teacher. In any case, Sudarshan's thesis was issued as a jointly authored University of Rochester preprint on September 16, 1957, the data of George's 26th birthday, just thirtyfour years ago. This evening I should like to recall those early years of close association with George Sudarshan and describe ever so briefly the pain and joy of a major scientific discovery.

I first met George Sudarshan thirty-eight years ago - in 1953 - in Bombay. In late August 1953, Hideki Yukawa organized the first international conference in theoretical physics after the end of the American occupation of Japan. Homi Bhabha, several years earlier, had been persuaded by Prime Minister Nehru of India to accept the dual responsibility of being director of the Tata Institute for Fundamental Research and Chairman of the Indian Atomic Energy Commission, headquartered in Bombay. Apparently, Bhabha kept a watchful eye on the proceedings of the Rochester conferences and noted that Maurice Lévy - with his Lévy potential [1] - was the "hero" of the latest Rochester conference (held in April 1953) [Robert Oppenheimer, who was chairman of the advisory committee for the early Rochester conferences made the

Reprint requests to Prof. Dr. G. Süssmann. "hero" judgment each year!]. Bhabha invited Lévy and me to lecture at the Tata Institute for the month preceeding the Japanese conference. The jet plane was still some years off so I met Maurice in Paris en route to India and our plane then made several refuelling stops - in Cairo, Dharaan, and Basra - before reaching hot and humid Bombay.

The drive from the Bombay Airport to the Taj Mahal Hotel, fronting directly on the Arabian Sea, was the beginning of a month-long culture shock for Lévy and myself in India. The Tata Institute was then located on the grounds of the former British Yacht Club, with the wine cellars converted to experimental labs and the street level entertainment rooms, with much open space and a glut of pigeons, providing office space for the theorists. Bhabha was a severe taskmaster and requested Lévy and me to lecture during the religious holidays (of which there are many in India during August), except for the Parsi holiday which he observed; this gave us a long weekend respite to visit the three most famous caves in India: Elephanta, Ellora and Ajanta, about which Nehru exults in his autobiography. I gave a series of lectures on the pionnucleon interaction and had the good fortune to have my lecture notes written up by a young Indian scientist, whose name at that time was E.C. George (a typical Syrian Christian surname from Kerala, I was told). [I am sure that most of you are aware that E. C. George was lengthened to E. C. George Sudarshan when George married Lalitha.]

Sudarshan spoke to me about coming to Rochester as a graduate student and, after checking his credentials with Bhabha, I informed him that he would be welcome once he fulfilled his commitments to Tata. It took two years for George to be released by the Tata Institute, during which time he had the good sense (or should I say prescience) to master several books on spinors. By the time George arrived in Rochester - in 1955 - the annual Rochester conference was in full swing, there were other excellent graduate students in the Rochester Physics Department (e.g. Okubo and 
Regge) and a goodly number of post-docs and visiting professors in particle physics in residence; all this helped to create, I believe, a stimulating environment for the talented young scientist from India.

But more than conferences and associates influenced Sudarshan's early career; a major turning point came in December 1956 with the discovery of parity violation. Sudarshan listened with rapt attention when the $\theta-\tau$ meson puzzle was discussed earlier at the Sixth Rochester Conference (in April 1956) - he even joined me in proposing a more conservative resolution of the $\theta-\tau$ puzzle than parity violation. I am sure that Sudarshan was as eager as I was to reexamine weak interaction theory when Madame Wu and her collaborators announced the large parity violation effect in the $\beta$ decay of $\mathrm{Co}^{60}$ [2]. I had been more than a casual participant in weak interaction developments ever since 1939 - when, in my thesis, I invoked the Gamow-Teller selection rule for the proton-proton reaction in order to pin down the internal temperature-density distribution in white dwarf stars [3]. Moreover, I was cognizant of the attempts by Tiomno and Wheeler [4] and others during the period 1947-49 - after the two-meson theory was confirmed by the $\pi \rightarrow \mu$ discovery - to ascertain whether a universal Fermi interaction (UFI) was operative among the weak interactions then known. It was therefore completely natural - after the Wu et al. announcement - to suggest to Sudarshan that he might take a fresh look at to whether a common Lorentz structure and strength could be assigned to all weak interactions. Sudarshan plunged into this problem with alacrity and exceeding good taste; he made a comprehensive analysis, within the framework of the UFI hypothesis, of the published and unpublished experiments in $\beta$ decay, $\mu$ decay, $\pi$ decay and $\mathrm{K}$ decay. I must remind you that during 1957 new parity-violating experiments were undertaken and old parity-conserving experiments were restudied at an incredible pace.

By the time of the Seventh Rochester Conference in April 1957, it was clear to both Sudarshan and mayself that the only possible UFI for weak processes was $\mathrm{V}-\mathrm{A}$ (with a lefthanded neutrino) and not a combination of $\mathrm{S}$ and $\mathrm{T}$ (with a righthanded neutrino), as was widely believed. While the $\beta$ decay data were confusing, muon decay mandated the $\mathrm{V}-\mathrm{A}$ choice and, moreover, an elegant chirality invariance argument applied to all hadron and lepton currents - patterned after the two-component neutrino hypothesis - uniquely predicted the universal $\mathrm{V}-\mathrm{A}$ theory. We were aware of four experiments contradicting $\mathrm{V}-\mathrm{A}$ : the two published parity-conserving experiments on the electronneutrino angular correlation in $\mathrm{He}^{6}$ (favoring the $\mathrm{T}$ interaction) and the substantial disagreement of the measured branching ratio in pion decay (to e and $\mu$ ) with the sharp A prediction of $1.3 \times 10^{-4}$. By April 1957 , there were also two preliminary parity-violating experiments (one reported in preprint form and the second as a private communication to me) - on the electron polarization from muon decay and the asymmetry from polarized neutron decay - that disagreed with the $\mathrm{V}-\mathrm{A}$ theory. We realized that if any one of these four experiments prevailed, one would have to abandon UFI or the underlying assumptions of a twocomponent neutrino and/or the law of conservation of leptons.

All this was interesting stuff and, in a rational world, it should have been reported at the Rochester conference in April 1957, which, after all, was my creation. Here I must confess to my first "cardinal blunder": the failure to arrange for a report on the $\mathrm{V}$-A theory during the course of the Seventh Rochester Conference. The explanation of my behavior in this instance is somewhat complicated and, looking back, was downright silly; the problem was that I had made a compact at that time with the Rochester Conference Advisory Committee to hold down the number of invited participants from throughout the world to approximately 150 . In return, it was agreed that graduate students from Rochester, of whom there were many, could attend as observers but not as full-fledged participants. That was the first problem; the second problem was that I was scheduled - at an early meeting of the Advisory Committee - to speak about some rather exciting results obtained at Rochester in connection with a semi-phenomenological two-nucleon potential program in the several hundred $\mathrm{MeV}$ region. Before my 1953 trip to Japan, a post-doc at Rochester, Chuck Oxley, had discovered (on the Rochester synchro-cyclotron) an unexpectedly large double-scattering effect in proton-proton elastic scattering at $150 \mathrm{MeV}$ [5]. The Oxley et al. results contradicted the predictions of the Lévy potential, which was the outcome of the most sustained quantum field-theoretic attempt (up to that date) to deduce the two-nucleon potential from the pseudoscalar pion-nucleon interaction. The Lévy potential gave reasonably good agreement for single two-nucleon scattering up to $150 \mathrm{MeV}$ but was bad for double scattering. I started to look into this discrepancy with Lévy during my Bombay 
visit and then undertook a major program with Okubo and other graduate students at Rochester to determine the general structure of the two-nucleon potential in the intermediate energy region. Several months before the Seventh Rochester Conference, one of my graduate students, Pete Signell, produced a beautiful fit (the so-called Signell-Marshak potential) of the existing single and double two-nucleon elastic scattering experiments in the several hundred $\mathrm{MeV}$ region [6]. And, hence, when the Seventh Rochester Conference began, I was obliged to report at length on the two-nucleon interaction and it seemed necessary since I was sensitive to the possible charge of "hogging" the sessions - to make another arrangement for reporting the $\mathrm{V}-\mathrm{A}$ theory. Paul Matthews, who was visiting professor at Rochester that year and fully conversant with the $\mathrm{V}-\mathrm{A}$ results, agreed to make suitable remarks during one of the discussion periods.

So far, these two excuses seem plausible to me thirty-four years after the act but I must still explain why I did not speak up when Matthews failed to intervene during two splendid opportunities provided by the review talks of T. D. Lee and C. S. Wu [7]. The strong message of Lee and Wu at the Seventh Rochester Conference was threefold: (1) the Columbia $\mathrm{He}^{6}$ experiment had settled the question of the Gamow-Teller part of the $\beta$ interaction in favor of $\mathrm{T}$ (rather than $\mathrm{A}$ ) [8]; (2) since the muon interaction is V (and A), UFI, according to Lee, "cannot be realized in the way we have expressed it"; and (3) a new experiment by Wu on the positron asymmetry from $\mathrm{Co}^{58}$ raised doubts about the presence of $\mathrm{S}$ rather than $\mathrm{V}$ (in addition to $\mathrm{T}$ ) in the $\beta$ interaction. The basic conclusion to be drawn from the Lee and Wu talks was that the $\beta$ interaction was probably $\mathrm{V}$ and $\mathrm{T}$, so that one would be forced to assign opposite helicities to the neutrinos emitted in the Fermi- and Gamow-Teller-type $\beta$ transitions $\left(v_{L}\right.$ for $\mathrm{V}$ and $v_{R}$ for $\mathrm{T}$ ), a very displeasing prospect indeed.

To add to the confusion, the possibility of a V, T $\beta$ interaction was reinforced by two rumors circulating at the Seventh Rochester Conference: one rumor was that Felix Boehm at Cal Tech had obtained a similar result to that of $\mathrm{Wu}$ for $\mathrm{Co}^{58}$, and the second rumor was that an Illinois group had measured the electron-neutrino angular correlation from $\mathrm{A}^{35}$ (a dominantly Fermi transition) and was finding a $\mathrm{V}$ instead of an S interaction [9]. It looked very much as if the $\beta$ interaction was a combination of $\mathrm{V}$ and $\mathrm{T}$, after all, and that UFI would have to be abandoned. This combination of circumstances put a crimp in my de- sire to bring up the universal V-A theory at the Seventh Rochester conference and was probably the reason why Matthews never spoke up. I cannot explain why I did not, as a minimum, ask whether the $\mathrm{He}^{6}$ experiment had been repeated elsewhere, except to acknowledge that there was a halo surrounding the Columbia $\beta$ decay program at the time and that $\mathbf{I}$ partly succumbed to the prevalent view of the correctness of this crucial experiment. I know that this is a lame excuse, George, but I must tell this friendly audience about two further mistakes on my part before I ask forgiveness for my three "cardinal blunders".

While I was reluctant to argue at the April 1957 Rochester conference for $\mathrm{V}-\mathrm{A}$ as the UFI option, as long as a consistent picture did not emerge from the parity-violating experiments, I knew that I would be presenting a paper on our $\mathrm{V}-\mathrm{A}$ theory at the forthcoming Pauda-Venice Conference in September, and I expected to clarify the V, T situation with Boehm shortly after my arrival at the Rand Corporation in California in mid-June (to take up a short-term consultantship). I told Sudarshan about my plans, and we agreed that he could continue on the Rochester graduate student payroll as long as he continued working on the $\mathrm{V}-\mathrm{A}$ theory while in California. This arrangement permitted us to meet with Boehm and to complete both the abstract and paper promised Professor N. Dallaporta, Chairman of the Padua-Venice Conference. Apart from the muddled priority question that arose later, I still believe that this arrangement was reasonable since it allowed time for certain key $\beta$ decay experiments to pass from rumor to completion stage and thereby to consolidate the experimental underpinning of the $\mathrm{V}-\mathrm{A}$ theory.

Be that as it may, a few days after I arrived in Santa Monica in mid-June of 1957 - to take up my duties at the Rand Corporation - I ran into Gell-Mann who was also a part-time consultant. Instead of talking about classified Rand business, I told Gell-Mann about the $\mathrm{V}-\mathrm{A}$ theory and asked him, if he would be so kind to set up an early luncheon meeting with his colleague, Boehm, so that Sudarshan and I could receive an update on whether the $\mathrm{V}, \mathrm{T}$ combination was a mirage insofar as the parity-violating $\beta$ decay experiments were concerned. The luncheon meeting took place during the first week of July and was attended by Boehm, Sudarshan, Gell-Mann, Berthold Stech (who was then a postdoc at $\mathrm{Cal}$ Tech), and myself. At this meeting, Boehm informed us that his most recent measurement in $\mathrm{Sc}^{46}$ of the same asymmetry parameter 
he had measured in $\mathrm{Co}^{58}$ [10], was consistent with a $\mathrm{V}, \mathrm{A} \beta$ interaction (with a lefthanded neutrino) or an $\mathrm{S}, \mathrm{T} \beta$ interaction (with a righthanded neutrino); this was sufficient reassurance for Sudarshan and myself, and our abstract for the $\mathrm{V}$-A paper was sent to Dallaporta in Padua, and the paper completed, within a matter of days. Gell-Mann claims in his 1983 Catalunya (Spain) talk on "Particle theory from S matrix to quarks" that, at the "summit" luncheon meeting, he mentioned the $\mathrm{V}-\mathrm{A}$ theory as a possible "last stand"; however, that claim does not jibe with the recollections of the other four participants in the "summit" meeting - Boehm, Stech, Sudarshan and myself. Gell-Mann's Catalunya remarks also do not jibe with Feynman's account of the genesis of the Feynman-Gell-Mann version of $\mathrm{V}-\mathrm{A}$ theory. Incidently, Stech was very quiet at the "summit" meeting and when I inquired, years later, why he had not extended the chirality invariance principle - about which he had shown so much familiarity in his 1955 paper with Jensen [11] - to quickly opt for $\mathrm{V}-\mathrm{A}$ after parity violation was announced, he informed me that, as a Cal Tech post-doc in 1957, he had mentioned the idea to Gell-Mann who discouraged him from pursuing the chirality invariance approach.

After the "summit" meeting, I discharged my duties at the Rand Corporation, gave a seminar on the $\mathrm{V}-\mathrm{A}$ theory at Stanford and returned to Rochester in September, where I arranged for a belated preprint of our $\mathrm{V}$-A paper to be sent out, as I have said, on September 16 (the date of George's 26th birthday) prior to its presentation to the Padua-Venice Conference during the last week of September. I recall telling the Padua audience that the $\mathrm{V}-\mathrm{A}$ theory required the "murder" of four experiments (including the $\mathrm{He}^{6}$ experiment) and overhearing - during the intermission - Jack Steinberger whisper to Leon Lederman that Marshak was "nuts" (or something to that effect) to question the sacrosanct $\mathrm{He}^{6}$ experiment performed by the Columbia group. Whatever the skeptism of Steinberger and others about the $\mathrm{V}-\mathrm{A}$ theory in Padua, Sudarshan and I were pushing hard for the redoing of the four experiments because the stakes were so high. As you all know, the four experiments, whose demise was required by the $\mathrm{V}-\mathrm{A}$ theory, were all redone during the next two years, and the new results were in complete accord with the $\mathrm{V}-\mathrm{A}$ theory. The most striking additional confirmation of the $\mathrm{V}-\mathrm{A}$ theory came in 1958 with the direct measurement of the lefthanded helicity of the neutrino in the ingenious experiment on
$\mathrm{K}$ capture in $\mathrm{Eu}^{152}$, performed by Maurice Goldhaber and collaborators at Brookhaven [12], for which Goldhaber just won the Wolf Prize.

The drive to test the universal $\mathrm{V}-\mathrm{A}$ theory of weak interactions gathered further momentum after September 1957, when the Feynman-Gell-Mann paper began to circulate in preprint form. By coincidence, their preprint was dated September 16, the same date as ours, but their preprint was en route to rapid publication in Phys. Rev. (January 1958) [13] whereas our paper was processed slowly as part of an unexpectedly large Proceedings of the Padua-Venice Conference [14] and only appeared in May 1958, where it was buried midst the great outpouring of papers at the Padua-Venice Conference. Some persons have asked me why we did not also publish our $\mathrm{V}$-A paper in a journal where it would receive wider attention, and my admittedly sheepish reply has been that I did not believe in "double" publication, particularly when a preprint was sent out. This turned out to be my second "cardinal blunder" against the interests of my junior colleague, George Sudarshan, who was in no position to fight back in those early days. Bethe underlines the gravity of this ineptitude on my part in an interview with Jagdish Mehra and Charles Weiner in 1966, when he says "unfortunately, Marshak and Sudarshan did not write it up as a paper for a normal journal ... nobody ever bothers to read the proceedings of a conference afterwards, and I think this was just bad luck". The reprinting of our Padua-Venice paper in P. K. Kabir's edited book "The Development of Weak Interaction Theory" [15] did not seem to help matters much in this regard.

Several incidents during the late fall of 1957 and early 1958 made me realize that I had indeed been too puristic in refraining from the "double" publication in journal and proceedings so widely practiced by highly reputable colleagues. The first shock came when I attended a December 1957 Advisory Committee meeting at the Institute for Advanced Study to come up with our recommendations of subjects and speakers for the Eighth Rochester Conference to be held in CERN in 1958. Before we started our deliberations, Oppenheimer enthused about the new $\mathrm{V}-\mathrm{A}$ theory of Feynman and Gell-Mann as I listened in stunned surprise (since Oppie had received our $\mathrm{V}$-A preprint in September and had told me in October - during a Washington encounter - that he preferred our chirality-invariant argument for $\mathrm{V}-\mathrm{A}$ to the two-component Klein-Gordon approach of the Feynman-Gell-Mann 
preprint). Nine years later - shortly before his death Oppie rediscovered our Padua-Venice paper and wrote me: "It is a beautiful paper and, for whatever good it is, even at this late date I read it with excitement and great pleasure" and, indeed, he asked Tullio Regge (who was at the Institute then and with whom he was lunching rather frequently at the end) to tell me how sorry he was that he had not paid more attention to our original $\mathrm{V}-\mathrm{A}$ paper.

Returning to the months after the Padua-Venice Conference, a second chastening experience occurred in January 1958, at the time of the APS meeting, which impelled me to commit what I call my third "cardinal blunder" in the matter of the $\mathrm{V}-\mathrm{A}$ theory. Feynman had been invited to speak on his $\mathrm{V}-\mathrm{A}$ theory with Gell-Mann and told a large APS audience in his typical bravado fashion how he had been inspired in Brazil to construct the $\mathrm{V}-\mathrm{A}$ theory and that several experiments had to be "killed" to validate the theory. I could not resist subjecting myself to the embarassment of remarking during the discussion period - in the presence of my former professor, Hans Bethe, who was chairing the session - that I had regaled a smaller audience at the Padua-Venice Conference the previous September with equal enthusiasm about our $\mathbf{V}-\mathbf{A}$ theory and had listed four experiments that had to be "murdered" in order for the theory to stand. Dick Feynman's reply was that he was aware that Sudarshan and I had first proposed the $\mathrm{V}$ - A theory but did not know what Gell-Mann would say. Again, as in the case of Oppenheimer, Feynman tried to make amends in later years; Dick wrote me a letter in 1985, in which he said: "It was great seeing you and talking. I hope some day we can get this straightened out and give Sudarshan the credit for priority that he justly deserves ... these matters all vex me - and I wish I had not caused you and Sudarshan such discomfort. At any opportunity I shall try to set the record straight as I have always done - but nobody believes me when I am serious".

The turn of events during the months after the Padua-Venice Conference greatly saddened George Sudarshan and led to my committing the third "cardinal blunder". I proposed to Sudarshan that we publish a short note in Phys. Rev. on "Chirality Invariance and the Universal Fermi Interaction" in order to reiterate the chirality invariance framework for our version of the $\mathrm{V}-\mathrm{A}$ theory (in contradistinction to the Feynman-Gell-Mann version) and to take stock of experimental developments favoring the $\mathrm{V}-\mathrm{A}$ theory since the Padua-Venice Conference. Our short note (published in March 1958 [16]) was not intended as a substitute for our detailed 1957 Padua-Venice paper but, unfortunately, it was treated by all too many physicists in later years as the sole publication of our V-A theory. Indeed, even gracious Dick Feynman, who faithfully stuck to the facts on the priority question, apparently never read our original Padua-Venice paper but only our short note. How else explain his statement as "Summarizer" at the Neutrino 1974 conference [17], in which he said: "We have a conventional theory of weak interactions invented by Marshak and Sudarshan, published by Feynman and Gell-Mann, and completed by Cabibbo - I call it the conventional theory of weak interactions - the one which is described as the V-A theory".

Well, George, I have recounted the three "cardinal blunders" that your well-intentioned professor committed in the aftermath of your fundamental scientific discovery. I do express my deep regrets for the pain caused by these decisions on my part during the hectic year 1957-58. I sincerely hope that the recital of these miscalculations - on the occasion of your 60th birthday celebration - and your forgiveness of them, will purge your soul (and mine) of the pain associated with the $\mathbf{V}-\mathbf{A}$ theory and enable you to remember with undiluted intellectual joy the triumphs of the $\mathrm{V}-\mathrm{A}$ theory. I remind you of the excitement generated by the redoing, after the Padua-Venice Conference, of each of the four experiments contradicting the $\mathrm{V}-\mathrm{A}$ theory, culminating in the first correct measurement at CERN of the branching ratio for charged pion decay. On this point, I can do no better than quote from Igor Tamm's summary talk at the 1959 Rochester Conference in Kiev [18], in which he said: "I wish first of all to point out the brilliant success of weak $\mathrm{V}-\mathrm{A}$ interaction theory now fully established by experimental research ... I believe that the $\mathrm{V}-\mathrm{A}$ interaction theory occupies an exceptional place in the history of our science: when this theory was first proposed, it contradicted many experimental facts which seemed to be quite reliable, but, within only two years, it was found that all the experiments contradicting the theory were erroneous ...".

George, your mastery of Cartan's "Leçons sur les Spineurs" before you left Bombay for Rochester in 1955 served you well and you should take great pride and satisfaction in the fact that the chirality invariance derivation of the $\mathrm{V}-\mathrm{A}$ theory in our original paper (and your thesis) has withstood the test of time, in 
contrast to the two-component Klein-Gordon derivation of the Feynman-Gell-Mann version of $\mathbf{V}-\mathbf{A}$, which is field-theoretically sick and has been discarded. As people are becoming increasingly aware, chirality invariance is crucial for the standard electroweak group, chiral gauge anomalies fix the chiral quark and lepton flavor quantum numbers in the standard model, and chiral fermions place strong restrictions on all theories attempting to "go beyond the standard model".

The joy of discovery of the $\mathrm{V}-\mathrm{A}$ theory - independent of the method of derivation - is best communicated by our departed friend, Dick Feynman, in conversations with Jagdish Mehra in January 1988, shortly before his death; I quote [19]:

"As I thought about it, as I beheld it in my mind's eye, the goddam thing was sparkling, it was shining brightly! As I looked at it, I felt that it was the first

[1] M. M. Lévy, Phys. Rev. 88, 72 (1952).

[2] C. S. Wu, E. Ambler, R. Hayward, D. Hoppes and R. Hudson, Pys. Rev. 105, 1413 (1957).

[3] R. E. Marshak, Ap. J. 92, 321 (1940).

[4] J. Tiomno and J. A. Wheeler, Rev. Mod. Phys. 21, 153 (1949).

[5] C. L. Oxley et al, Phys. Rev. 19, 419 (1953).

[6] P. Signell and R. E. Marshak, Phys. Rev. 106, 832 (1957).

[7] T. D. Lee, Proc. of Seventh Rochester Conf. on High Energy Phys., 1957, p. VIII-7; C. S. Wu, ibid, p. VII-22.

[8] B. M. Rustad and S. L. Ruby, Phys. Rev. 97, 991 (1995).

[9] B. Hermannsfeldt, D. Maxson, P. Stahelin and J. Allen, Phys. Rev. 107, 641 (1957).

[10] F. Boehm and A. H. Wapstra, Phys. Rev. 109, 456 (1958).

[11] B. Stech and J. D. Jensen, Zeits. f. Phys. 141, 175 (1955).

[12] M. Goldhaber, L. Grodzins and A. Sunyar, Phys. Rev. 109, 1015 (1958). time, and the only time in my scientific career, that I knew a law of nature that no one else know. Now, it wasn't as beautiful a law as Dirac's or Maxwell's but my new equation for $\beta$ decay was a bit like that. It was the first time that I discovered a new law, rather than finding a more efficient method of calculating from someone else's theory ... I learned later that others had thought of it at about the same time or a little before, but that didn't make any difference. At the time I was doing it, I felt all the thrill of a new discovery! ... I thought now I have completed myself."

George, this is a tremendous, albeit indirect, tribute from one of the great scientists of our era and should assuage the pain and enhance the joy of your having been involved in a major scientific discovery in your early career, at the tender age of 26 .

[13] R. P. Feynman and M. Gell-Mann, Phys. Rev. 109, 193 (1958).

[14] E. C. G. Sudarshan and R. E. Marshak, Proc. of PaduaVenice Conference on "Mesons and Newly Discovered Particles", 1957, p. V-14.

[15] E. C. G. Sudarshan and R. E. Marshak in The Development of Weak Interaction Theory, ed. by P. K. Kabir, Gordon and Breach, 1963, p. 118.

[16] E. C. G. Sudarshan and R. E. Marshak, Phys. Rev. 109, 1860 (1958).

[17] R. P. Feynman, Proc. of Intern. Conf. on Neutrino Physics and Astrophysics, (U. of Pennsylvania), p. 300.

[18] I. Tamm, Proc. of Ninth International Conference in High Energy Physics, Kiev, 1959, Vol. II, p. 412.

[19] Jagdish Mehra authorized this quote from his forthcoming book: The beat of a different drum: the life and science of Richard Phillips Feynman, to be published by Oxford University Press in 1992. 